\title{
Traumatismo encéfalocraneano, ¿factor de riesgo para esquizofrenia o causa de psicosis? A propósito de un caso.
}

\begin{abstract}
Traumatic brain injury: a risk factor for schizophrenia or a cause of psychosis? A case report.
María Teresa Rivera-Encinas ${ }^{1,2,4, a, b, c,}$, Joshep A. Revilla-Zúñiga a ${ }^{\text {3,4,a,c, }}$, Lizardo Cruzado ${ }^{2,4, a, d}$.

RESUMEN

El traumatismo encéfalo craneano (TEC) ha sido identificado como causa desencadenante de psicosis y también como factor de riesgo y antecedente en la eclosión de esquizofrenia. Con el fin de ilustrar el diagnóstico diferencial entre estas dos posibilidades, se presenta un caso cínico y se revisa brevemente la literatura pertinente. Paciente varón de 21 años, con antecedentes familiares de esquizofrenia, sufrió un TEC severo a los 13 años y dos años después empezó a presentar delusiones de daño y referencia y alucinaciones auditivas a las que siguieron poco después, síntomas de tipo negativo. El diagnóstico diferencial entre esquizofrenia paranoide y psicosis secundaria a TEC se decantó a favor de la primera. Se requiere abarcar la mayor cantidad de elementos clínicos (anamnésicos y de exploración física), exámenes auxiliares neuropsicológicos, imagenológicos y electroencefalográficos en este tipo de disquisición clínica pues muchas veces el diagnóstico dista de ser claro. La evolución clínica del trastorno, como es usual, puede dirimir el dilema diagnóstico.
\end{abstract}

PALABRAS CLAVE: Traumatismos craneocerebrales, trastornos psicóticos; esquizofrenia; factores de riesgo. (Fuente: DeCS Bireme).

\section{SUMMARY}

Traumatic brain injury (TBI) has been identified as a causal factor in the emergence of psychosis, and also as a risk factor preceding the development of schizophrenia. In order to illustrate the differential diagnosis between these two conditions, a case report and a brief review of pertinent literature are presented are presented. A 21-yearold male patient with a family history of schizophrenia, suffered of a severe TBI at age 13, and two years later started to present referential and persecutory delusions and auditory hallucinations, followed, shortly thereafter, by negative symptoms. The differential diagnosis between paranoid schizophrenia and psychosis secondary to TBI was decided in favor of the former. In a diagnostic disquisition of this type, it is necessary to include the largest number of clinical elements (anamnestic and physical examination-related), neuropsychological, neuroimaging and electroencephalographic, besides laboratory tests, as many times the diagnosis is far from clear. The clinical evolution of the disorder, as usual, can assist in the resolution of the diagnostic dilemma.

KEY WORDS: Craniocerebral trauma, psychotic disorders, schizophrenia, risk factors. (Source: MeSH NLM).

' Universidad de San Martín de Porres, Facultad de Medicina, Instituto de Investigación, Centro de Investigación en Salud Pública. Lima, Perú

2 Instituto Nacional de Salud Mental "Honorio Delgado - Hideyo Noguchi”. Lima, Perú.

3 Hospital Víctor Larco Herrera. Lima, Perú.

4 Facultad de Medicina Alberto Hurtado. Universidad Peruana Cayetano Heredia. Lima, Perú.

a Médico psiquiatra; ${ }^{b}$ Magíster en Salud Pública con mención en Salud Ocupacional; ${ }^{\text {c Docente; }}$

d Profesor auxiliar. 


\section{INTRODUCCIÓN}

Las enfermedades neuropsiquiátricas, en conjunto, son las responsables del mayor número de años de vida saludables perdidos por discapacidad en el Perú (1), y a nivel mundial también originan enorme carga de enfermedad (2). Dentro de las patologías mencionadas, los traumatismos encéfalocraneanos (TEC) son una causa de importante morbimortalidad, sobre todo en niños y adolescentes (3). Asimismo, en nuestro país la esquizofrenia representa, luego de la depresión y las demencias, la tercera causa de años perdidos por discapacidad dentro de este conjunto de padecimientos psiquiátricos y neurológicos (1).

El TEC en la actualidad se define, de forma estándar, como la alteración de la función cerebral producto de la aplicación de una fuerza externa contra la cabeza y que deviene en una o más de las siguientes consecuencias: disminución en el nivel de conciencia o confusión, amnesia, anomalías neurológicas o neuropsicológicas objetivas o lesiones intracraneales (4). Asimismo, se ha tipificado la severidad del TEC en base a determinados parámetros tales como los hallazgos de neuroimágenes, la duración de la amnesia postraumática o la duración de la pérdida de conciencia, y la puntuación de la escala de Glasgow (5). Grosso modo, el $80 \%$ de los TEC son leves, el 10\%, moderados, y el 10\%, graves; el 10\% de los primeros, el $65 \%$ de los segundos y el $99 \%$ de los últimos, desarrollará algún tipo de alteración neurológica o psiquiátrica, si el paciente sobrevive al evento (6).

Existe desde épocas inmemoriales el conocimiento empírico de que los golpes en la cabeza podrían posteriormente asociarse a diversos problemas mentales. Dentro de las principales consecuencias psiquiátricas del TEC -sin mencionar las estrictamente neurológicas- se enumeran la depresión, ansiedad y trastorno de estrés postraumático, psicosis, y otros desórdenes menos específicos $(7,8)$. En la literatura científica, la relación entre psicosis y lesión cerebral traumática ha sido y es objeto de diferentes controversias. En la actualidad, los hallazgos de investigación parecen haberse decantado en dos configuraciones: el TEC como factor de riesgo indirecto pero asociado al posterior desarrollo de esquizofrenia y otras enfermedades psiquiátricas primarias (9) y, por otro lado, el TEC como factor causal directo para el consiguiente desarrollo de un cuadro de psicosis postraumática por daño cerebral directo (10).
Con la finalidad de discernir entre estos dos modelos causales, presentamos un caso clínico que resulta ilustrativo de esta disquisición etiológica y diagnóstica.

\section{Caso Clínico}

Paciente varón de 21 años, natural y procedente de Lima, soltero, con secundaria completa, con antecedentes paternos de una tía y una prima con esquizofrenia. El padre era alcohólico. A los 13 años fue atropellado por una motocicleta y sufrió un TEC severo, presentó convulsión tónico-clónica generalizada luego de tres horas y estuvo 22 horas en estado de coma. Permaneció 9 días hospitalizado. Al salir de alta asistió a controles con el neurólogo sin novedad aparente aunque se le notaba algo enlentecido y más callado que lo usual. Retomó sus estudios escolares y tuvo bajo rendimiento académico por problemas en la concentración y memoria. No presentó más convulsiones.

A la edad de 15 años empezó a experimentar desrealización y la sensación pasajera de ser observado cuando salía a la calle, por esto permanecía en casa lo más posible. Su rendimiento escolar comenzó a declinar por dificultades de concentración y de memoria, experimentaba bloqueos del pensamiento y además ideas obsesivas de contaminación y chequeo. Pese a estas dificultades, terminó su educación secundaria a los 17 años. Luego aparecieron delusiones dismorfofóbicas centradas en su rostro, lo que redundó en mayor aislamiento. Solo recibió tratamiento dermatológico.

Alos 19 años de edad, por persistir la referencialidad, abandonó sus estudios universitarios apenas iniciados y volvió a recluirse en casa. Aparecieron delusiones persecutorias y se agravaron las dismorfofóbicas. Tenía alucinaciones auditivas de la voz de una cantante norteamericana hablándole, afirmaba además que ella le controlaba la mente, podía saber todo lo que él pensaba y le había otorgado poderes. Tenía soliloquios y risas inmotivadas, a veces se mostraba triste y hasta lloroso pero otras muy irritado y colérico. Permanecía meses enteros realizando mínimas tareas domésticas con prominentes abulia y aplanamiento afectivo.

Pocas semanas antes de su última atención, presentó agitación psicomotriz tras sufrir un leve accidente de tránsito: acusó a la cantante norteamericana de haber querido asesinarlo y rompió varios enseres 
Traumatismo encéfalocraneano, ¿factor de riesgo para esquizofrenia o causa de psicosis? A propósito de un caso.

en su domicilio. En la evaluación se le halló hostil, desaliñado, delusivo, alucinado, disgregado y sin conciencia de enfermedad. Se le hizo el diagnóstico de esquizofrenia paranoide. Se le inició tratamiento antipsicótico con $6 \mathrm{mg}$ diarios de risperidona (hasta una dosis de $8 \mathrm{mg} /$ día) más $400 \mathrm{mg}$ de carbamazepina, pero la respuesta fue escasa. Debido a esto, se le agregó haloperidol hasta una dosis de $10 \mathrm{mg} /$ día, sin embargo los síntomas persistieron sin mayores cambios. En la resonancia magnética de encéfalo se halló "área encefalomalácica cortical temporal derecha y en la encrucijada parieto- temporal del mismo lado". El electroencefalograma fue informado como anormal por presentar actividad lenta frontal.

Dada su escasa respuesta clínica, se decidió el cambio de antipsicótico a clozapina y se retiró además la carbamazepina. Con una dosis de $400 \mathrm{mg}$. de clozapina se ha logrado una mejoría parcial pero progresiva, sobre todo de los síntomas positivos.

\section{DISCUSIÓN}

El estudio de las psicosis postraumáticas depende en gran medida de la definición que establezcamos de estos dos conceptos (psicosis y traumatismo craneal, antes hemos enunciado la clasificación estandarizada de TEC, que es relativamente reciente). Ya Kraepelin insinuó en el siglo XIX una posible asociación entre el TEC y el subsecuente desarrollo de psicosis y esquizofrenia (11). Los primeros trabajos sobre psicosis postraumáticas abarcaban no solo cuadros estrictamente psicóticos sino también con características de otra índole como problemas amnésicos, trastornos afectivos, deterioros cognitivos diversos y cambios conductuales secuelares inespecíficos (12). Así, la amplia revisión de Davison y Bagley, que recogía casos incluso desde 1870, consideraba series con una incidencia de psicosis postraumática alta, cercana a $10 \%$. Los trabajos posteriores de Achté et al., hallaron incidencias de psicosis desde $8,9 \%$ y hasta $26 \%$. Estas cifras elevadas pueden explicarse, además de los diagnósticos heterogéneos considerados, por los diferentes periodos de seguimiento que, en algún caso, han llegado hasta las cinco décadas. Es decir, la diversidad de criterios involucrados tanto en la demarcación diagnóstica como en los límites de seguimiento asumidos válidos, tornó brumosa la demarcación de cuándo un TEC podía considerarse como causa del inicio de un cuadro psicótico y cuándo constituir mero factor de riesgo para cuadros psicóticos bien delimitados como la esquizofrenia (12). Antes de comentar la investigación más reciente, debe acotarse, como apuntan Bernal y Vega, que es muy difícil establecer una relación causaefecto directa entre el TEC y el trastorno psiquiátrico en todos los casos (7). Además, existe la posibilidad de que el TEC actúe como desencadenante o facilitador de la expresión de otras vulnerabilidades relacionadas con los trastornos psiquiátricos primarios (8). Pero, a priori, se ha descartado que se trate de una mera asociación al azar de dos eventos no demasiado infrecuentes (TEC y psicosis) (13).

Una salvedad necesaria es reconocer la distinción clínica de un cuadro psicótico como tal respecto a los cuadros inmediatamente consecutivos al trauma en los que, debido a la prominente alteración de conciencia, pueden aparecer fenómenos como ideas delusivas y alucinaciones, a veces acompañados de agitación y confusión mental, pero que propiamente deben catalogarse como cuadros de delirium y de ninguna manera como episodios de psicosis (14).

En general, los estudios más recientes cifran la aparición de cuadros de psicosis entre un 1 a $10 \%$ del total de pacientes que sufren TEC (15). El TEC incrementa el riesgo de desarrollo de episodios psicóticos hasta en 2 a 3 veces respecto a la población general. Los factores de riesgo para la aparición de psicosis postraumática son el sexo masculino, problemas neuropsiquiátricos previos, afectación de lóbulos temporales o frontales, mayor severidad de la lesión, epilepsia o deterioro cognitivo postraumático, abuso de sustancias psicoactivas y la presencia de antecedentes familiares de esquizofrenia (16). En adición, la psicosis secundaria a TEC difiere de las psicosis psiquiátricas primarias en la edad de inicio, algunas características del perfil sintomático y hallazgos imagenológicos específicos. Un desafío permanente en la caracterización de la psicosis postraumática es que la relación temporal entre ambos eventos (trauma e inicio de psicosis) puede no ser inmediatamente evidente. Algunos autores han señalado que el intervalo menor de un año entre ambos eventos se asocia a lesión cerebral difusa mientras que lapsos mayores se asociarían a daños localizados sobre todo en el lóbulo temporal (16). En la Tabla 1 pueden apreciarse datos diagnósticos a favor de una psicosis postraumática respecto a una psicosis primaria.

La literatura más reciente sobre el tema distingue dos cuadros psicóticos postraumáticos: el trastorno de tipo delusional y el trastorno "tipo esquizofrénico" (se asume que no es propiamente una esquizofrenia) (10). El primero se caracteriza por la presencia únicamente 
Tabla 1. Datos clínicos que orientan a favor de una psicosis postraumática respecto a una psicosis psiquiátrica primaria.

\footnotetext{
- Edad de inicio tardío (más de 35 años)

- Menor periodo de latencia entre TEC e inicio de síntomas

- Inexistencia de síntomas previos al TEC

- Nulos o escasos rasgos previos de personalidad esquizotípica, esquizoide o paranoide

- Menor frecuencia o inexistencia de desorganización del pensamiento o catatonía

- Menor frecuencia o inexistencia de síntomas negativos

- Nulos o escasos antecedentes familiares de esquizofrenia

- Presencia de síntomas cognitivos secuela del TEC

- Presencia de otros trastornos psiquiátricos secundarios al TEC

- Menor duración de la sintomatología psicótica

- Menor duración necesaria de tratamiento antipsicótico o respuesta más rápida

- Mejor evolución clínica de la psicosis, sin deterioro o mínimo deterioro.
}

Elaborado utilizando las fuentes: 11,13,16

de delusiones, sobre todo de tipo Capgras (delusión de que las personas familiares son reemplazadas por impostores idénticos) y paramnesia reduplicativa (fenómeno en que el paciente afectado cree que un lugar físico existe simultáneamente en dos o más localizaciones) (17). Los síntomas negativos son escasos $(25 \%)$ pero deben distinguirse de la apatía y abulia secundarias al TEC en sí, lo que no suele ser sencillo. La mayoría de casos (64\%) ocurren antes de transcurrido un año del traumatismo. Por otro lado, el trastorno psicótico semejante a esquizofrenia implica la presencia de alucinaciones (más frecuentemente auditivas aunque puede haber también visuales) además de las delusiones, estas últimas suelen ser persecutorias. Los síntomas negativos son más frecuentes que en el trastorno de tipo delusional antes mencionado: se hallan en más de $40 \%$ de los casos (10). Los síntomas de desorganización del pensamiento y catatónicos son bastante raros (13). Asimismo, en cuanto al tiempo de demora entre el traumatismo y el inicio del trastorno de tipo esquizofrénico, se registra un lapso mayor, con una media de 3,3 años entre la lesión y el inicio de la psicosis, aunque en otras series puede prolongarse hasta 4 o 5 años (10). Debe anotarse que la serie de la que emergen estos hallazgos es de menos de 100 pacientes reportados en diferentes fuentes. Otros datos que pueden ayudar a la distinción entre psicosis por TEC y esquizofrenia, en cuanto a exámenes auxiliares de imágenes y electroencefalografía, se pueden apreciar en la tabla 2: la psicosis por TEC difiere de la esquizofrenia en el hallazgo de lesiones más circunscritas y específicas.

Aunque no hay acuerdo unánime, no se ha corroborado la relación entre severidad del trauma y desarrollo de psicosis por cuanto luego de traumas de distinta intensidad, de leves a severos, se ha verificado el desarrollo de cuadros psicóticos (10). Es probable que esto se deba a la interposición de factores subyacentes de vulnerabilidad (p. ej. antecedentes familiares de esquizofrenia $u$ otros). Otro dato a tener en cuenta en el diagnóstico diferencial es la psicosis secundaria a una epilepsia postraumática pues las convulsiones como consecuencia del TEC son frecuentes (18).

Por otro lado, se han desarrollado estudios epidemiológicos cuya naturaleza es muy distinta a las series de casos clínicos que hemos mencionado. El más amplio, de un registro poblacional danés de 113906 personas que tuvieron atención hospitalaria por TEC, explora la asociación entre este evento y el posterior diagnóstico de enfermedad mental (19). Aquí se halló que el TEC se asocia a un incremento de riesgo de 1,65 veces para esquizofrenia, 1,59 para depresión y 1,28 para trastorno bipolar. Predeciblemente el mayor riesgo es para el diagnóstico de trastorno psiquiátrico orgánico (incremento de 4,39 veces). Asimismo este estudio señala que el mayor incremento de riesgo se verificó durante el primer año después de la lesión y, sobre todo, si el TEC sucedió entre los 11 y 15 años de edad (19). Este efecto etario podría subrayar una edad particularmente sensible del neurodesarrollo para el desarrollo posterior de enfermedades mentales.

Previamente, se habían ejecutado otros dos grandes estudios epidemiológicos: el de Harrison et al. con 785 052 pacientes seguidos durante 7 años, y en el que no se halló asociación entre TEC y esquizofrenia bajo ningún parámetro estudiado (20). Por otra parte, Nielsen et al., ejecutaron un diseño de casos y controles que incluyó 8288 pacientes seguidos durante 15 años: aquí se halló un leve incremento de riesgo de esquizofrenia 
Traumatismo encéfalocraneano, ¿factor de riesgo para esquizofrenia o causa de psicosis? A propósito de un caso.

Tabla 2. Comparación de hallazgos de exámenes auxiliares entre Psicosis por TEC y Esquizofrenia.

\begin{tabular}{|c|c|c|}
\hline & Psicosis por TEC & Esquizofrenia \\
\hline $\begin{array}{c}\text { Hallazgos positivos (\%)* } \\
\text { RMN / TAC }\end{array}$ & 55 & $12-35$ \\
\hline Atrofia cerebral (\%) & 0 & $12-35$ \\
\hline Anormalidades focales (\%) & 89 & $6-9$ \\
\hline Lesiones evidenciables (\%) & $\begin{array}{l}34 \text { Frontal } \\
19 \text { Temporal }\end{array}$ & $\begin{array}{c}\text { Atrofia frontal, temporal } \\
\text { hipocampal, de ganglios basales y } \\
\text { talámica }\end{array}$ \\
\hline Dilatación ventricular (\%) & 19 & $>50$ \\
\hline $\begin{array}{l}\text { Hallazgos positivos (\%) } \\
\text { SPECT / PET }\end{array}$ & $\begin{array}{l}100 \\
86 \text { temporal } \\
14 \text { frontal }\end{array}$ & $\begin{array}{c}20 \text { - } 60 \text { Hipoperfusión frontal, } \\
\text { temporal, talámica y de ganglios } \\
\text { basales }\end{array}$ \\
\hline \multirow[t]{2}{*}{ EEG hallazgos positivos (\%) } & 87 & Normal \\
\hline & $\begin{array}{l}\text { Ondas en punta región } \\
\text { temporal }\end{array}$ & $\begin{array}{c}\text { Normal u Ondas lentas en región } \\
\text { frontal }\end{array}$ \\
\hline
\end{tabular}

* Por ejemplo: gliosis cicatricial, lesión axonal difusa, hematomas residuales, etc.

Modificado de (10)

tanto para la concusión craneana como para el TEC severo (21). Un metaanálisis que incluyó 172 estudios para explorar la relación entre TEC y esquizofrenia corroboró la presencia de un riesgo relativo de entre 1,65 a 2,8 para los expuestos a TEC; igualmente, el riesgo fue mayor para aquellos individuos con antecedentes familiares de esquizofrenia y no se pudo corroborar relación entre severidad del TEC y desarrollo de la enfermedad (22).

Es muy difícil suponer que ambos tipos de estudios (series de casos y estudios epidemiológicos) evalúen los mismos tipos de casos de psicosis (9). Es igualmente arduo sostener en base a la evidencia, la existencia por separado de dos fenómenos netamente diferenciados: psicosis postraumática por un lado y, por el otro, esquizofrenia con TEC como factor de riesgo (23). Existe sin duda un amplio grupo intermedio en que coalescen formas mixtas como resultado de los diferentes factores patogenéticos involucrados (15). Además debe reconocerse un sesgo adicional: la mayoría de TEC que reciben atención y se registran son los graves y, en menor cuantía, los moderados y leves, aunque estos sean más frecuentes. Esto hace más desafiante estructurar la distinción entre el TEC como factor de riesgo para esquizofrenia o como causa de psicosis postraumática: la definición epistémica de estos factores aún no posee la evidencia empírica suficiente para distinguir per se uno de otro: se requieren estudios prospectivos, comparativos y controlados para delinear mejor ambos cuadros $(23,24)$.
En relación a la farmacoterapia, se recomienda igualmente el uso de antipsicóticos para la esquizofrenia con antecedente de TEC así como para la psicosis secundaria a TEC. El uso de anticonvulsivantes será valorado de acuerdo a la clínica que presente cada paciente. Debe tenerse en cuenta la comorbilidad con problemas convulsivos y el bajo umbral convulsivo por retraso en la recuperación neuronal en las primeras semanas a meses después del TEC. Junto a estas consideraciones, la clínica de labilidad afectiva, impulsividad, irritabilidad o agresividad, aconsejará el uso de anticonvulsivantes junto al antipsicótico que es la base del tratamiento (25).

En nuestro caso, el inicio del cuadro psicótico aconteció dos años luego del TEC. No puede soslayarse en la consideración diagnóstica el importante antecedente familiar de esquizofrenia y la presencia creciente de síntomas negativos, así como el hallazgo de ondas lentas frontales en el electroencefalograma: todo esto apunta al diagnóstico de esquizofrenia, aparentemente precipitada en su inicio por el TEC sufrido, pese a que en el estudio de resonancia magnética cerebral el hallazgo más prominente fue la lesión antigua del TEC. Consideramos que la esquizofrenia de nuestro paciente habría empezado aun sin el estímulo patogénico del TEC.

En conclusión, la disquisición diagnóstica de una psicosis por TEC y un cuadro de esquizofrenia no es sencilla y deben valorarse una serie de factores como el tiempo de inicio luego del TEC, los antecedentes 
familiares de esquizofrenia, la presencia de síntomas negativos y de desorganización, los hallazgos de imagen, neuropsicología y electroencefalografía además de otros factores adicionales. La trascendencia del pronóstico del cuadro de psicosis diagnosticada amerita el esfuerzo y permitirá medidas adicionales en el tratamiento específico y rehabilitación respectiva.

\section{Correspondencia:}

Lizardo Cruzado

Instituto Nacional de Salud Mental "Honorio Delgado - Hideyo Noguchi".

Jr. Eloy Espinoza 709. Urb. Palao. San Martín de Porres. Lima 15102. Lima, Perú.

Correo electrónico: lizardo.cruzado.d@upch.pe

\section{REFERENCIAS BIBLIOGRÁFICAS}

1. Ministerio de Salud. Carga de enfermedad en el Perú. Estimación de los años de vida saludables perdidos 2012. Lima: Ministerio de Salud; 2014.

2. Whiteford HA, Ferrari AJ, Degenhardt L, Feigin V, Vos T. The global burden of mental, neurological and substance use disorders: An analysis from the global burden of disease Study 2010. PLoS One. 2015; 10(2): e0116820.

3. Guillén-Pinto D, Zea A, Guillén-Mendoza D, Situ M, Reynoso C, Milla L, et al. Traumatismo encefalocraneano en niños atendidos en un hospital nacional de Lima, Perú 2004-2011. Rev Perú Med Exp Salud Pública. 2013; 30: 630-634.

4. Menon DK, Schwab K, Wright DW, Maas AI. Position statement: definition of traumatic brain injury. Arch Phys Med Rehabil. 2010; 91: 1637-1640.

5. Castaño-Monsalve B. Características clínicas y evoluciónpsicopatológica deloscambios conductuales secundarios a traumatismo craneoencefálico. Tesis doctoral. Departamento de Psiquiatría, Universidad Autónoma de Barcelona; 2013. (Fecha de acceso: 15 febrero del 2018). Disponible en: http://www. tdx.cat/bitstream/handle/10803/133263/bcm1de1. pdf; sequence $=1$

6. Pérez AF, Agudelo VH. Trastornos neuropsiquiátricos por trauma craneoencefálico. Rev Colomb Psiquiat. 2007; 36 (Supl 1): 40S-51S.

7. Bernal-Pacheco O, Vega-Rincón M, HernándezPreciado JF. Consecuencias neuropsiquiátricas del trauma craneoencefálico. Rev Fac Med. 2009; 17: 6574.

8. Castaño-Monsalve B, Bernabeu-Guitart M, López R, Bulbena-VilasarA, Quemada JI. Perfil psicopatológico de pacientes con traumatismo craneoencefálico evaluados mediante el Inventario Neuropsiquiátrico. Rev Psiquiatr Salud Ment. 2012; 5:160-166.

9. Kim E. Does traumatic brain injury predispose individuals to develop schizophrenia? Curr Opin Psychiatry. 2008; 21: 286-289.

10. Fujii D, Ahmed I. Psychotic disorder caused by traumatic brain injury. Psychiatr Clin N Am. 2014; 37: 113-124.

11. Wolkin A, Malaspina D, Perrin M, McAllister TW, Corcoran C. Psychotic disorders. En: Silver JM, McAllister TW, Yudofsky SC. (Eds.) Textbook of traumatic brain injury. Arlington: American Psychiatric Publishing; 2011. pp. 189-197.

12. Quemada JI, Mimentza N, González E. Psicosis y trauma craneal. En: Fernández LJ, Berrios G, Yáñiz B (eds.) Las psicosis atípicas o transitorias: De la epistemología al tratamiento. Madrid: UNED; 2012. pp. 129-146.

13. Sachdev P, Smith JS, Cathcart S. Schizophrenia-like psychosis following traumatic brain injury: a chartbased descriptive and case-control study. Psychol Med. 2001;31:231-239.

14. Trzepacz PT, Kean J, Kennedy RE. Delirium and posttraumatic confusion. En: Silver JM, McAllister TW, Yudofsky SC. (Eds.) Textbook of traumatic brain injury. Arlington: American Psychiatric Publishing; 2011. pp. 145-171.

15. Batty R, Rossell SL, Francis AJP, Ponsford J. Psychosis following traumatic brain injury. Brain Impair. 2013;14:21-41.

16. Bhalerao S, Geurtjens C, Thomas G, Kitamura CR, Zhou C, Marlborough M. Understanding the neuropsychiatric consequences associated with significant traumatic brain injury. Brain Inj. 2013; 27: 767-774.

17. Politis M, Loane C. Reduplicative Paramnesia: a review. Psychopathology. 2012; 45:337-343.

18. Arciniegas DB, Harris SN, Brousseau KM. Psychosis following traumatic brain injury. Int Rev Psychiatry. 2003; 15: 328-340.

19. Orlovska S, Pedersen M, Benros M, Mortensen P, Agerbo E, Nordentoft M. Head injury as risk factor for psychiatric disorders: A nationwide register-based follow-up study of 113906 persons with head injury. Am J Psychiatry. 2014; 171: 463-9.

20. Harrison G, Whitley E, Rasmussen F, et al. Risk of schizophrenia and other nonaffective psychosis among individuals exposed to head injury: case control study. Schizophr Res. 2006; 88 (1-3):119126.

21. Nielsen AS, Mortensen PB, O'Callaghan E, et al. Is head injury a risk factor for schizophrenia? Schizophr Res. 2002; 55 (1-2):93-98. 
Traumatismo encéfalocraneano, ¿factor de riesgo para esquizofrenia o causa de psicosis? A propósito de un caso.

22. Molloy C, Conroy RM, Cotter DR, Cannon M. Is traumatic brain injury a risk factor for schizophrenia? A meta-analysis of case-controlled population-based studies. Schizophr Bull. 2011; 37: 1104-1110.

23. Guerreiro DF, Navarro R, Silva M, et al. Psychosis secondary to traumatic brain injury. Brain Inj. 2009; 23:358-361.

24. van Reekum R, van Reekum EA. Traumatic brain injury and psychosis. Psychiatr Times. 2018; 35: 27-

29. (Fecha de acceso: 15 de febrero del 2018)
Disponible en: http://www.modernmedicine.com/ sites/default/files/images/digital/PSY/psy0118_ ezine.pdf

25. Chew E, Zafonte RD. Pharmacological management of neurobehavioral disorders following traumatic brain injury. A state-of the-art review. J Rehabil Res Dev. 2009;46: 851-879.

Recibido: $13 / 06 / 2018$

Aceptado: 27/11/2018 